TITLE:

\title{
Direct observation of geometric phases using a three-pinhole interferometer
}

\section{AUTHOR(S):}

Kobayashi, H.; Tamate, S.; Nakanishi, T.; Sugiyama, K.; Kitano, M.

\section{CITATION:}

Kobayashi, H....[et al]. Direct observation of geometric phases using a three-pinhole interferometer. PHYSICAL REVIEW A 2010, 81(1): 012104.

\section{ISSUE DATE:}

2010

URL:

http://hdl.handle.net/2433/148384

RIGHT:

(c) 2010 The American Physical Society 
PHYSICAL REVIEW A 81, 012104 (2010)

\title{
Direct observation of geometric phases using a three-pinhole interferometer
}

\author{
H. Kobayashi, ${ }^{1}$ S. Tamate, ${ }^{1}$ T. Nakanishi,,${ }^{1,2}$ K. Sugiyama, ${ }^{1,2}$ and M. Kitano ${ }^{1,2}$ \\ ${ }^{1}$ Department of Electronic Science and Engineering, Kyoto University, Kyoto 615-8510, Japan \\ ${ }^{2}$ CREST, Japan Science and Technology Agency, Saitama 332-0012, Japan
}

(Received 21 October 2009; published 7 January 2010)

\begin{abstract}
We present a method to measure the geometric phase defined for three internal states of a photon (polarizations) using a three-pinhole interferometer. From the interferogram, we can extract the geometric phase related to the three-vertex Bargmann invariant as the area of a triangle formed by interference fringes. Unlike the conventional methods, our method does not involve the state evolution. Moreover, the phase calibration of the interferometer and the elimination of the dynamical phase are not required. The gauge invariance of the geometric phase corresponds to the fact that the area of the triangle is never changed by the local phase shift in each internal state.
\end{abstract}

DOI: 10.1103/PhysRevA.81.012104

PACS number(s): 03.65.Vf, 03.65.Ta, 07.60.Ly

\section{INTRODUCTION}

When a quantum system evolves in time and returns to its initial state, the final and initial wave functions can differ by a phase factor composed of two parts: a dynamical phase proportional to the time integral of the instantaneous energy and a geometrical phase, which depends only on the path traced in the ray space and not on the energy and the rate of evolution. The geometric phase was originally discovered by Berry [1] in the adiabatic and cyclic evolution of a pure quantum state. Since then it has been generalized to nonadiabatic evolution $[2,3]$ and noncyclic evolution [4,5]. Its applications in practical fields such as fault-tolerant quantum computation [6,7] and weak measurement [8] are also proposed.

Most approaches for observing the geometric phase, such as the interferometric and polarimetric methods [9-15], require the state evolution. Note that the geometric phase induced by such evolution appears as the global phase factor, which cannot be measured directly. Therefore in order to observe the geometric phase, we must prepare the reference state, that is, the state that is left unevolved, and measure the relative phase between the evolved and the reference states. To remove additional phase shifts associated with the aforementioned operations, phase calibration is required [16]; that is, the relative phase must be determined by comparing the cases with and without the state evolution. Moreover, the dynamical phase must be eliminated from the relative phase. These two considerations, namely, the phase calibration and the elimination of the dynamical phase, lead to experimental complications.

On the other hand, based on the quantum kinematic approach [17], the geometric phase can be attributed to the geometric structure of the Hilbert space, and not to the state evolution. In particular, an important consequence of the kinematic approach has been to show the close relationship between the geometric phase and the Bargmann invariant [18]. The $n$-vertex Bargmann invariant is a complex quantity that is determined by $n$ points in the ray space. The phase of the $n$-vertex Bargmann invariant is identical to the geometric phase for a closed ray-space curve obtained by connecting the corresponding $n$ states with geodesics. The geometric phase for a smooth curve can be obtained from the Bargmann invariant by approximating a smooth curve with a chain of geodesics. In this sense, the phase of the Bargmann invariant, particularly that of the three-vertex Bargmann invariant, is the primitive building block of the geometric phase.

The purpose of this article is to report a method to measure the geometric phase or the three-vertex Bargmann invariant without the state evolution. All we need to do is to prepare the three states and let them interfere directly. In our method, the phase calibration and elimination of the dynamical phase are not required. As shown in Fig. 1, we assign three states, $\left|\psi_{j}\right\rangle(j=1,2,3)$, to the internal states of the photon from three pinholes and obtain a three-pinhole interferogram, which contains three distinct interference fringes due to each pinhole pair. Using a certain data processing, we can extract the geometric phase directly from the interferogram.

\section{GEOMETRIC PHASE AND BARGMANN INVARIANT}

In 1956, Pancharatnam [19] introduced the definition of the phase relation between any two (nonorthogonal) states. Assume that $\left|\psi_{1}\right\rangle$ and $\left|\psi_{2}\right\rangle$ are two different states and that $\left|\psi_{1}\right\rangle$ is exposed to the $\mathrm{U}(1)$ shift $\mathrm{e}^{\mathrm{i} \phi}$. By superimposing two such states, we have the intensity $I \propto 1+$ $\left|\left\langle\psi_{1} \mid \psi_{2}\right\rangle\right| \cos \left(\phi+\arg \left\langle\psi_{1} \mid \psi_{2}\right\rangle\right)$. The interference fringes are shifted by $\arg \left\langle\psi_{1} \mid \psi_{2}\right\rangle$, the relative phase between two states. In particular, when they constructively interfere or $\arg \left\langle\psi_{1} \mid \psi_{2}\right\rangle=$ $0,\left|\psi_{1}\right\rangle$ and $\left|\psi_{2}\right\rangle$ are said to be in-phase.

A remarkable feature of this relation is its nontransitivity; even if $\left|\psi_{1}\right\rangle$ is in-phase with $\left|\psi_{2}\right\rangle$ and $\left|\psi_{2}\right\rangle$ with $\left|\psi_{3}\right\rangle$, the relative phase between $\left|\psi_{1}\right\rangle$ and $\left|\psi_{3}\right\rangle$ is, in general, not zero. It is easy to show that the nonzero phase difference between $\left|\psi_{1}\right\rangle$ and $\left|\psi_{3}\right\rangle$ can be written as

$$
\begin{aligned}
\Delta_{3}\left(\psi_{1}, \psi_{2}, \psi_{3}\right) & =\arg \left\langle\psi_{1} \mid \psi_{2}\right\rangle\left\langle\psi_{2} \mid \psi_{3}\right\rangle\left\langle\psi_{3} \mid \psi_{1}\right\rangle \\
& =\sum_{(i, j)_{\text {cyclic }}} \arg \left\langle\psi_{i} \mid \psi_{j}\right\rangle,
\end{aligned}
$$

where $(i, j)_{\text {cyclic }} \equiv(1,2),(2,3),(3,1) . \quad \Delta_{3}$ is called the Pancharatnam phase [19]. The product $\left\langle\psi_{1} \mid \psi_{2}\right\rangle\left\langle\psi_{2} \mid \psi_{3}\right\rangle\left\langle\psi_{3}\right.$ $\left|\psi_{1}\right\rangle$ is the Bargmann invariant [18] for the three states. Equation (1) is gauge invariant, that is, independent of the choice of the local phase factor of each state because the bra and ket vectors for each state appear in a pair. The phase $\Delta_{3}$ is the primitive building block of the geometric phase based on the quantum kinematic approach [17]. It turns out that 


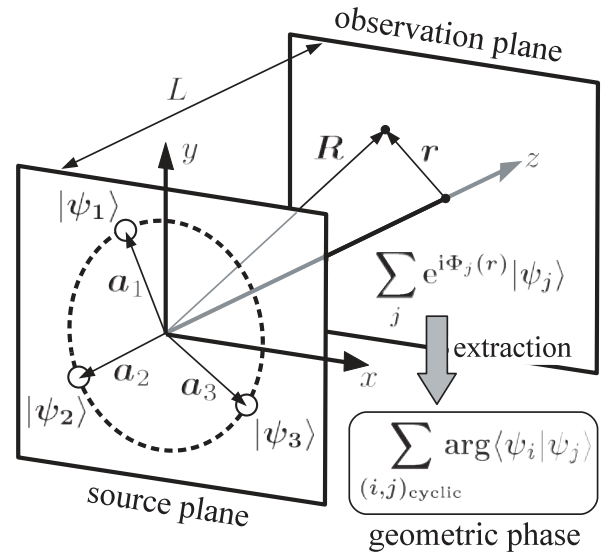

FIG. 1. Coordinate system of three-pinhole interferometer. The three pinholes are irradiated by monochromatic light and the three spherical waves interfere. We can extract the geometric phase for three states, $\left|\psi_{1}\right\rangle,\left|\psi_{2}\right\rangle$, and $\left|\psi_{3}\right\rangle$, from the three-pinhole interferogram.

$\Delta_{3}$ is simply related to the geometric phase associated with the geodesic triangle in the ray space. For a two-state system such as the polarization of a photon, $\Delta_{3}$ is proportional to the solid angle of the spherical triangle on the Bloch sphere with vertices at $\left|\psi_{1}\right\rangle,\left|\psi_{2}\right\rangle$, and $\left|\psi_{3}\right\rangle[19,20]$.

\section{GEOMETRIC PHASE AND RIDGE LINES}

As shown in Fig. 1, consider three pinholes irradiated by monochromatic light that has the wave number $k$. Without loss of generality, we may consider that the three pinholes are located at $\boldsymbol{a}_{j}(j=1,2,3)$ on the source plane $z=0$ with their origin at the circumcenter of the triangle formed by the three pinholes, and these vectors have the same length $a$ (see Fig. 1). The state of each photon from the pinholes is composed of two parts: the spatial part that is represented by the spherical wave and the internal state of the photon, namely, the polarization state. Assuming that the transmission probabilities of the three pinholes are the same for simplicity, the state on an observation plane at a distance of $L$ is represented by [21]

$$
|\Psi(\boldsymbol{r})\rangle=C \sum_{j=1}^{3} \frac{\mathrm{e}^{\mathrm{i}\left(k\left|\boldsymbol{R}-\boldsymbol{a}_{j}\right|+\phi_{j}\right)}}{\left|\boldsymbol{R}-\boldsymbol{a}_{j}\right|}\left|\psi_{j}\right\rangle
$$

where $\boldsymbol{R}$ is the position vector on the observation plane $z=L ; \boldsymbol{r} \equiv \boldsymbol{R}-\left(\boldsymbol{R} \cdot \boldsymbol{e}_{z}\right) \boldsymbol{e}_{z}$, the transverse component of $\boldsymbol{R}$ with the unit vector $\boldsymbol{e}_{z}$ along the $z$ axis; $C$, the dimensionless normalization constant; $\phi_{j}$, the phase of the $j$ th source; and $\left|\psi_{j}\right\rangle$, the polarization state of the $j$ th source. $\left|\boldsymbol{R}-\boldsymbol{a}_{j}\right|$ is the distance from the $j$ th source to a given observation point $\boldsymbol{R}$.

We make a paraxial approximation in the far-field regime, and moreover, we assume the stronger condition, $\left|\boldsymbol{R}-\boldsymbol{a}_{j}\right| \ll$ $\left(L^{3} / k\right)^{1 / 4} \ll L$. Under these assumptions, the spherical wave function in Eq. (2) is approximated as

$$
\frac{\mathrm{e}^{\mathrm{i} k\left|\boldsymbol{R}-\boldsymbol{a}_{j}\right|}}{\left|\boldsymbol{R}-\boldsymbol{a}_{j}\right|} \sim \frac{1}{L} \exp \left[\mathrm{i} k\left(L+\frac{r^{2}+a^{2}}{2 L}-\frac{\boldsymbol{r} \cdot \boldsymbol{a}_{j}}{L}\right)\right]
$$

where $r \equiv|\boldsymbol{r}|$. Therefore, the intensity distribution of the interference field, $p(x, y)$, can be written as

$$
\begin{aligned}
p(x, y) & =\||\Psi(\boldsymbol{r})\rangle \|^{2} \\
& =\frac{C^{2}}{L^{2}}\left\{-3+\sum_{(i, j)_{\text {cyclic }}} P_{i j}(x, y)\right\},
\end{aligned}
$$

with

$$
\begin{aligned}
P_{i j}(x, y)= & 2\left(1+\left|\left\langle\psi_{i} \mid \psi_{j}\right\rangle\right|\right. \\
& \left.\times \cos \left[\boldsymbol{k}_{i j} \cdot \boldsymbol{r}-\phi_{i j}+\arg \left\langle\psi_{i} \mid \psi_{j}\right\rangle\right]\right),
\end{aligned}
$$

where $\boldsymbol{k}_{i j} \equiv k\left(\boldsymbol{a}_{i}-\boldsymbol{a}_{j}\right) / L$ and $\phi_{i j} \equiv \phi_{i}-\phi_{j}$. Equation (5) corresponds to the double-slit interference fringe between the two states, $\left|\psi_{i}\right\rangle$ and $\left|\psi_{j}\right\rangle$ [19]. Therefore, the three-pinhole interferogram (4) contains three sets of interference fringes with different directions.

In order to extract the geometric phase from the total interferogram (4), we should focus our attention on the phase of the interference fringes $P_{i j}$, since their visibility $\left|\left\langle\psi_{i} \mid \psi_{j}\right\rangle\right|$ includes no information about the geometric phase in Eq. (1). Here, we consider the phase condition to attain the maximum of each interference fringe in Eq. (5),

$$
\boldsymbol{k}_{i j} \cdot \boldsymbol{r}=\phi_{i j}-\arg \left\langle\psi_{i} \mid \psi_{j}\right\rangle+2 n_{i j} \pi,
$$

where $n_{i j}$ are integers. Equation (6) defines three distinct sets of parallel lines, which we call ridge lines, on the observation plane $z=L$. The area $S$ of the triangle formed by the three distinct ridge lines, which we call a ridge triangle, is calculated as

$$
S=\frac{L^{2}}{4 k^{2} S_{0}}\left\{\Delta_{3}\left(\psi_{1}, \psi_{2}, \psi_{3}\right)-2 n \pi\right\}^{2},
$$

where $n=n_{12}+n_{23}+n_{31}$ and $S_{0}$ is the area of the triangle formed by the three pinholes. Equation (7) shows three important features of a ridge triangle. First, the area of the ridge triangle is essentially related to the geometric phase $\Delta_{3}$. In particular, we call a ridge triangle that includes no ridge lines inside as elemental. Assuming $0 \leqslant \Delta_{3}<2 \pi$, ridge triangles with $n=0$ and 1 in Eq. (7) are elemental. The area with $n=0$ is proportional to the square of the geometric phase $\Delta_{3}$ and that with $n=1$ is proportional to the square of $2 \pi-\Delta_{3}$. Second, we should note that the area $S$ does not depend on the choice of the local phases $\phi_{i}$, because the geometric phase $\Delta_{3}$ is gauge invariant. By introducing a phase shift to one of the pinholes, two sets of ridge lines are displaced but the areas of the ridge triangles are conserved. Third, any geometry of the three pinholes can form the ridge triangle related to the geometric phase since Eq. (7) is proportional to the square of the geometric phase regardless of the vectors $\boldsymbol{a}_{i}$.

\section{EXTRACTION OF RIDGE LINES}

A straightforward method to determine the ridge lines is the observation of individual interference fringes $P_{i j}(x, y)$ in Eq. (5) by closing one of the three pinholes. However, instead of using the three interferograms $P_{i j}(x, y)$, we can extract all the ridge lines from a single-shot interferogram $p(x, y)$ with the three pinholes. First, we introduce the 
vectors

$$
\boldsymbol{b}_{i} \equiv \boldsymbol{e}_{z} \times\left(\boldsymbol{a}_{j}-\boldsymbol{a}_{k}\right)=\frac{L}{k} \boldsymbol{e}_{z} \times \boldsymbol{k}_{j k},
$$

where $(i, j, k)=(1,2,3),(2,3,1),(3,1,2)$. The vector $\boldsymbol{b}_{i}$ on the observation plane $z=0$ is determined only from the geometry of the three pinholes. Considering the directional derivative along $\boldsymbol{b}_{i}$, we can eliminate one of the interference fringes, $P_{j k}(x, y)$, from the total interferogram $p(x, y)$ since $\boldsymbol{b}_{i}$ is orthogonal to $\boldsymbol{k}_{j k}$. The other two fringes remain as sinusoidal functions. In addition, by applying another directional derivative along $\boldsymbol{b}_{j}$, we can isolate the oscillation term of $P_{i j}(x, y)$ from $p(x, y)$ as

$$
\begin{aligned}
& \left(\boldsymbol{b}_{i} \cdot \nabla\right)\left(\boldsymbol{b}_{j} \cdot \nabla\right) p(x, y) \\
& \quad \propto\left\langle\psi_{i} \mid \psi_{j}\right\rangle \mid \cos \left[\boldsymbol{k}_{i j} \cdot \boldsymbol{r}-\phi_{i j}+\arg \left\langle\psi_{i} \mid \psi_{j}\right\rangle\right] .
\end{aligned}
$$

Then, a set of the ridge lines can be retrieved from Eq. (9). Examples are shown in Fig. 3. Interferograms for the three pinholes are shown in Fig. 3(a) and the three sets of ridge lines thus extracted are shown in Fig. 3(b).

As a result, we can determine the pure geometric phase instantaneously as the square root of the area of the ridge triangle extracted directly from the three-pinhole interferogram for three arbitrary states.

\section{EXPERIMENTS}

Our experimental setup is shown in Fig. 2. The light source is a 532-nm green laser. The source illuminates a thin copper foil that is perforated with three 0.1 -mm-radius pinholes forming an equilateral triangle of side length $1.5 \mathrm{~mm}$. At a distance of approximately $2 \mathrm{~m}$ from the three pinholes, the interfering patterns are captured using a charge-coupled

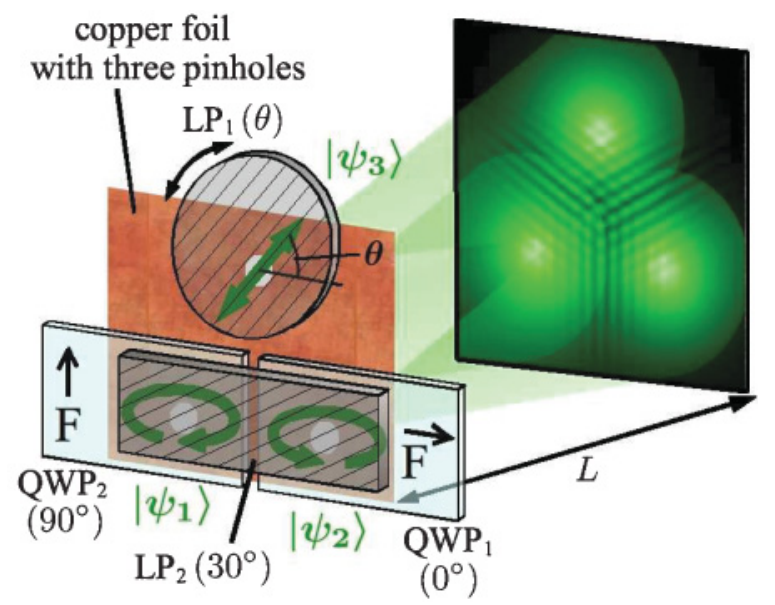

FIG. 2. (Color online) Experimental setup for three-pinhole interference with different polarization states. In front of the upper pinhole, we placed a film-type linear polarizer, $\mathrm{LP}_{1}$, attached to a rotatable mount with graduated scales for adjusting the angle $\theta$, whereas in front of the lower left and right pinholes we placed film-type quarter-wave plates having orthogonal fast axes, $0^{\circ}\left(\mathrm{QWP}_{1}\right)$ and $90^{\circ}\left(\mathrm{QWP}_{2}\right)$, respectively, behind a linear polarizer $\mathrm{LP}_{2}$ with a fixed angle of $30^{\circ}$. Incident light on the pinholes is circularly polarized, and transmittance of light through each pinhole is $50 \%$. Under this configuration, the visibility of the fringe $P_{i j}(x, y)$ is more than or equal to 0.5 , which is sufficient to retrieve clear ridge lines. device (CCD) camera. The CCD camera has an image resolution of $640 \times 480$ pixels, with the size of each pixel being $9 \mu \mathrm{m} \times 8 \mu \mathrm{m}$. According to the setting of polarization elements in Fig. 2, the polarization states from the left, right, and upper pinholes are $\left|\psi_{1}\right\rangle=(\sqrt{3}|\mathrm{H}\rangle+\mathrm{i}|\mathrm{V}\rangle) / 2,\left|\psi_{2}\right\rangle=$ $(\mathrm{i} \sqrt{3}|\mathrm{H}\rangle+|\mathrm{V}\rangle) / 2$, and $\left|\psi_{3}\right\rangle=\cos \theta|\mathrm{H}\rangle+\sin \theta|\mathrm{V}\rangle$, where $|\mathrm{H}\rangle$ and $|\mathrm{V}\rangle$ are the horizontal and vertical polarization states, respectively.

On a Poincaré sphere, $\left|\psi_{1}\right\rangle$ and $\left|\psi_{2}\right\rangle$ are both located at a latitude of $\pm 60^{\circ}$ on the prime meridian, and $\left|\psi_{3}\right\rangle$ is located on the equator at a longitude of $2 \theta$, which can be varied according to the setting of $\mathrm{LP}_{1}$. The geometric phase is proportional to the solid angle $\Omega$ of the spherical triangle formed by $\left|\psi_{1}\right\rangle$, $\left|\psi_{2}\right\rangle$, and $\left|\psi_{3}\right\rangle$ on the Poincare sphere; that is, $\Delta_{3}=-\Omega / 2$. It is calculated as

$$
\Delta_{3}\left(\psi_{1}, \psi_{2}, \psi_{3}\right)=\tan ^{-1}\left(\frac{1}{\sqrt{3}} \tan \theta\right),
$$

which moves between 0 and $2 \pi$ with respect to $\theta$.

Figure 3 shows our experimental results. Figure 3(a) shows the experimentally obtained interferograms for several values of $\theta$, and Fig. 3(b) shows the ridge lines extracted from the above interferograms. The shaded triangles in Fig. 3(b) are the elemental ridge triangles $(n=0)$ and the area of

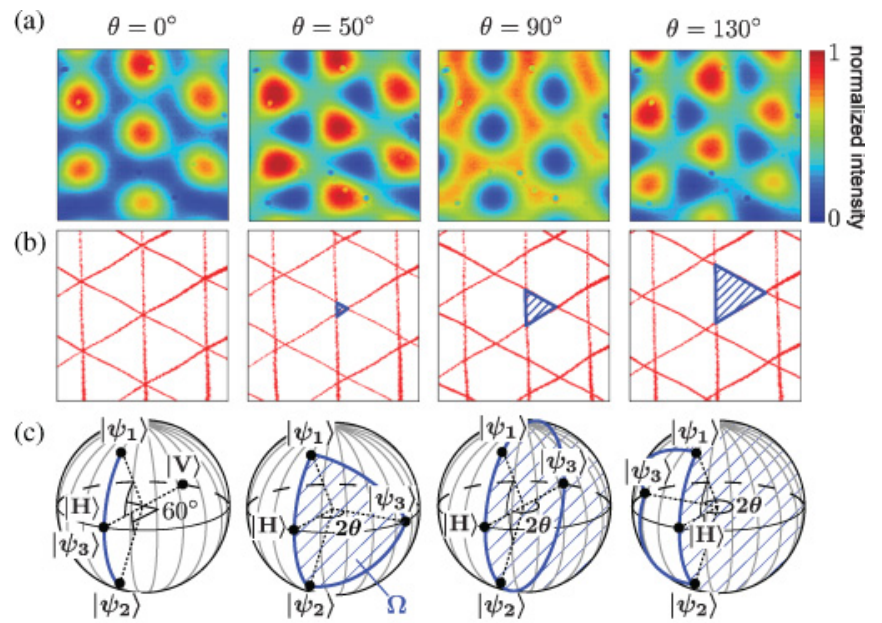

(d)

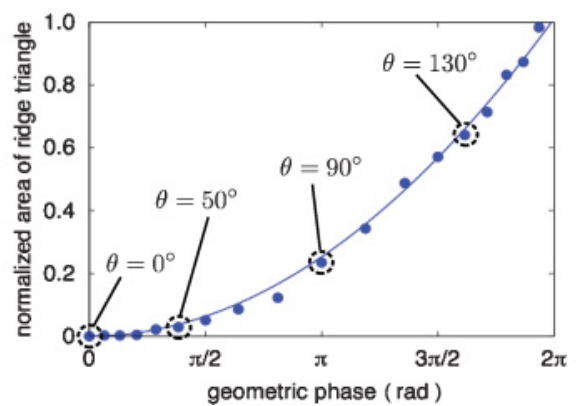

FIG. 3. (Color online) Ridge triangles and geometric phases for $\theta=0^{\circ}, 50^{\circ}, 90^{\circ}$, and $130^{\circ}$. (a) The three-pinhole interferogram obtained in our experiment. The actual size of each figure is $1.5 \mathrm{~mm} \times 1.5 \mathrm{~mm}$. (b) Ridge lines extracted from the above interferograms. (c) Corresponding spherical triangle on the Poincaré sphere. (d) Geometric phase versus area of ridge triangle. The area of the ridge triangle is normalized by the maximum area. The solid line indicates the theoretical curve, and it shows a quadratic characteristic. 


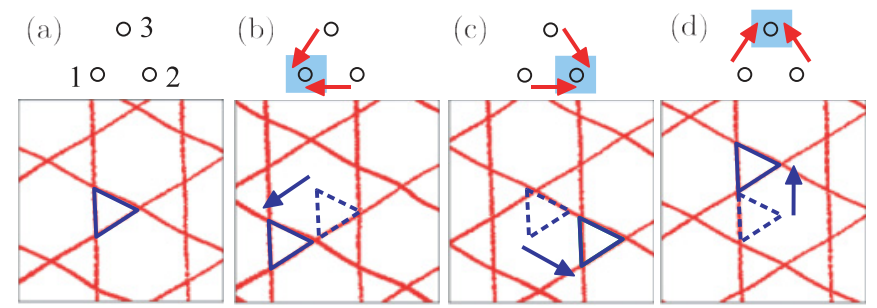

FIG. 4. (Color online) Ridge triangles for $\theta=90^{\circ}$ with (a) no shifts, (b) shift on pinhole 1, (c) shift on pinhole 2, and (d) shift on pinhole 3. The ridge triangle is parallel displaced, but it is not deformed for a local phase shift. The actual size of each figure is $1.5 \mathrm{~mm} \times 1.5 \mathrm{~mm}$.

the ridge triangle varies with the spherical triangle on the Poincaré sphere [see Fig. 3(c)]. The relationship between the elemental ridge triangle and the geometric phase is quantitatively analyzed in Fig. 3(d), in which the area of the elemental ridge triangle normalized by the maximum area is plotted as a function of the geometric phase calculated from Eq. (10). The solid line in Fig. 3(d) is the theoretical curve calculated from Eq. (7), and the experimental results [dots in Fig. 3(d)] are found to agree well with the theoretical prediction. In Fig. 3(b), we can also see the other elemental ridge triangle $(n=1)$, which is related to the complementary area on the Poincaré sphere $4 \pi-\Omega$.

Figure 4 shows variations of the ridge triangles when a local phase shift is introduced by inserting a thin (0.15-mm-thick) glass plate in front of each pinhole. Figure 4(a) shows the ridge lines without a phase shift as reference. When a phase shift is introduced at pinhole 1, as shown in Fig. 4(b), the two interference fringes $P_{12}(x, y)$ and $P_{31}(x, y)$ in Eq. (5) suffer the same phase shift and they are simultaneously displaced toward pinhole 1 . Thus, the ridge triangle is only parallel displaced along the ridge line of fringe $P_{23}(x, y)$, but it is not deformed. Similarly, a phase shift applied to pinhole 2 and pinhole 3 has no influence on the size of the ridge triangle, as shown in Figs. 4(c) and 4(d), respectively. The fact that the ridge triangle is not deformed shows the gauge invariance of the geometric phase in our experiment.

\section{CONCLUSION}

We have shown a procedure for measuring the geometric phase without state evolution using a three-pinhole interferometer. From the interferogram, we can extract the primitive building block of the geometric phase $\Delta_{3}$ (phase of the three-vertex Bargmann invariant) as the area of the ridge triangle. Our experiment requires no procedures for phase calibration and elimination of the dynamical phase. The gauge invariance of the geometric phase corresponds to the fact that the ridge triangle is not deformed by the local phase shift. Moreover, by using a CCD video camera followed by image processing to extract the ridge lines, we can see the geometric phase in real time.

\section{ACKNOWLEDGMENTS}

We thank Yosuke Nakata for providing useful comments and suggestions. This research is supported by the global COE program "Photonics and Electronics Science and Engineering" at Kyoto University.
[1] M. V. Berry, Proc. R. Soc. London A 392, 45 (1984).

[2] Y. Aharonov and J. Anandan, Phys. Rev. Lett. 58, 1593 (1987).

[3] J. Anandan, Nature (London) 360, 307 (1992).

[4] J. Samuel and R. Bhandari, Phys. Rev. Lett. 60, 2339 (1988).

[5] A. Morinaga, A. Monma, K. Honda, and M. Kitano, Phys. Rev. A 76, 052109 (2007).

[6] P. Zanardi and M. Rasetti, Phys. Lett. A264, 94 (1999).

[7] L. M. Duan, J. Cirac, and P. Zoller, Science 292, 1695 (2001).

[8] S. Tamate, H. Kobayashi, T. Nakanishi, K. Sugiyama, and M. Kitano, New J. Phys. 11, 093025 (2009).

[9] H. Schmitzer, S. Klein, and W. Dultz, Phys. Rev. Lett. 71, 1530 (1993).

[10] P. G. Kwiat and R. Y. Chiao, Phys. Rev. Lett. 66, 588 (1991).

[11] J. Brendel, W. Dultz, and W. Martienssen, Phys. Rev. A 52, 2551 (1995).
[12] C. L. Webb, R. M. Godun, G. S. Summy, M. K. Oberthaler, P. D. Featonby, C. J. Foot, and K. Burnett, Phys. Rev. A 60, R1783 (1999).

[13] D. Suter, K. T. Mueller, and A. Pines, Phys. Rev. Lett. 60, 1218 (1988).

[14] J. C. Loredo, O. Ortíz, R. Weingärtner, and F. DeZela, Phys. Rev. A 80, 012113 (2009).

[15] H. Kobayashi, S. Tamate, T. Nakanishi, K. Sugiyama, and M. Kitano, e-print arXiv:quant-ph/0907.2289.

[16] E. Sjöqvist, Phys. Lett. A359, 187 (2006).

[17] N. Mukunda, Ann. Phys. 228, 205 (1993).

[18] V. Bargmann, J. Math. Phys. 5, 862 (1964).

[19] S. Pancharatnam, Proc. Ind. Acad. Sci. A 44, 247 (1956).

[20] P. K. Aravind, Opt. Commun. 94, 191 (1992).

[21] M. Born and E. Wolf, Principles of Optics, 7th ed. (Cambridge University Press, Cambridge, UK, 1999), Chap. 8.8, pp. 484-499. 